\title{
Contribution des produits de cueillette dans l'alimentation humaine. Cas de Detarium microcarpum
}

\author{
Amadou Malé Kouyaté (1), Patrick Van Damme (2), \\ Bruno De Meulenaer (3), Hamidou Diawara (4)
}

$(\mathrm{I}, 4)$ Institut d'Economie Rurale, Sikasso, Mali

(2) Laboratoire d'Agriculture Tropicale et Subtropicale et d'Ethnobotanique

Université de Gand, Belgique

(3) Laboratoire de Chimie Alimentaire, Université de Gand, Belgique

\begin{abstract}
Les fruits de Detarium microcarpum sont très prisés des populations maliennes en saison sèche chaude. Nous avons donc cherché à évaluer leurs valeurs alimentaires et propriétés physico-chimiques en fonction de la diversité pédoclimatique au Mali. Une enquête ethnobotanique sur les valeurs alimentaires a été menée auprès des ethnies bobofing, sénoufo et minianka au sud du Mali. Trente échantillons de fruits ont été analysés au laboratoire (goût, sucre alimentaire, protéines, matière sèche, vitamine $\mathrm{C}$ et $\mathrm{pH}$ ). Des corrélations ont été établies entre ces paramètres et les dimensions des fruits. Les résultats ont montré que les fruits de $\mathrm{D}$. microcarpum sont très utilisés dans l'alimentation humaine de la zone de recherche. Ils ont également montré que ces fruits peuvent constituer une source de protéines végétales et de sucre alimentaire. Les connaissances locales et les paramètres physico-chimiques des fruits de $\mathrm{D}$. microcarpum peuvent justifier la valorisation de cette plante.
\end{abstract}

Mots clés: Mali, valeur nutritionnelle, espèce sous-utilisée, utilisation traditionnelle, ethnobotanique

The fruits of Detarium microcarpum are very much appreciated during the hot dry season by Malian consumers. Therefore, we tried to assess their food values and physicochemical properties according to the pedoclimatic diversity in Mali. An ethnobotanical survey on the food values was carried out with the bobofing, senoufo and minianka ethnic groups in southern Mali. Thirty samples of fruits were analyzed in the laboratory for taste, food sugar, proteins, dry matter, vitamin C and $\mathrm{pH}$. Correlations were established between these parameters and fruit dimensions. Results show that the fruits of $D$. microcarpum are very much used for food. They also show that these fruits can constitute a source of plant proteins and sugar. The local knowledge and the physicochemical parameters of D. microcarpum fruits can justify further valorization and domestication of this species.

Key words: Mali, nutritional value, under utilized crop, traditional crop, ethnobotany 


\section{Caractéristiques biochimiques des fruits de Detarium microcarpum}

\section{Introduction}

Les pays du Sahel ont adopté un cadre stratégique de sécurité alimentaire durable dans une perspective de lutte contre la pauvreté. Pour y parvenir, un accent particulier est mis sur la création des conditions d'une sécurité alimentaire structurelle, fondée prioritairement sur la mobilisation systématique et durable des ressources alimentaires de la sous-région.

La situation nutritionnelle dans la plupart des pays sahéliens est également caractérisée par des niveaux élevés de malnutrition protéino-énergétique, et de carence en vitamines. Pour remédier à ces insuffisances, les populations rurales font recours aux produits de cueillette mais leur disponibilité reste très saisonnière en raison des conditions dynamiques naturelles de production.

Les plantes comestibles sauvages contribuent considérablement à la nutrition du monde rural ouest africain (Freiberger et al., 1998). Parmi les organes consommés, les fruits sont considérés comme des suppléments alimentaires importants dans la région sahélienne pour augmenter la qualité de l'alimentation journalière des populations rurales (Glew et al., 2005). Cependant, le niveau de connaissance et d'information du monde rural ne leur permet pas toujours de tirer le meilleur profit des produits de cueillette consommés.

Les études anthropologiques ont démontré que les fruits ont formé une partie importante de l'alimentation de l'être humain pendant des centaines de milliers d'années. Il est admis que les arbres fournissent des apports alimentaires importants qui ne sont pas toujours assurés par la production agricole classique (Sène, 2000). Au regard de cette situation, une évaluation des caractéristiques biochimiques de ces produits s'impose afin de contribuer à la sélection d'individus supérieurs.

Les recherches sur Detarium microcarpum Guill. Perr. ont été sollicitées par les femmes du sud du Mali, à travers la plate-forme de la Commission Régionale des Utilisateurs des Résultats de Recherche du sud du Mali en vue de parvenir à sa conservation et son utilisation durables. Cette commission est une instance composée d'organisations paysannes qui aide à définir l'agenda de la recherche agricole axée sur la demande sociale.

Detarium microcarpum est une espèce fruitière des savanes africaines (figure $\mathrm{I}$ ). Son fruit est une drupe qui se caractérise par une graine (plus rarement plusieurs graines) enfermée dans un endocarpe qui est entouré par un mésocarpe, juteux, parfois fibreux et protégé extérieurement par un épicarpe (Schmidt, 2000). Au Mali, l'échantillonnage des individus de D. microcarpum a permis d'identifier des descripteurs intéressants pour la caractérisation des feuilles, des fruits, de l'endocarpe et des graines pour des programmes futurs de sélection et d'amélioration génétique en vue de déboucher sur sa domestication. De plus, les résultats des observations phénologiques ont permis de planifier les périodes de récoltes des fruits et des feuilles en vue de conserver et d'utiliser durablement les ressources génétiques de D. microcarpum (Kouyaté, 2005). Des études réalisées au Burkina Faso ont montré que la teneur en phénol et en flavonoïde des fruits 
de D. microcarpum est plus élevée que celle d'Adansonia digitata, Ziziphus mauritiana, Ximenia americana et Lannea microcarpa. Les mêmes études ont révélé une activité anti-oxydante très forte au niveau des fruits de D. microcarpum (Lamien-Meda et al., 2008). Au regard de ces études, le présent travail sur les accessions de $\mathrm{D}$. microcarpum au Mali est une première et vient compléter la littérature.

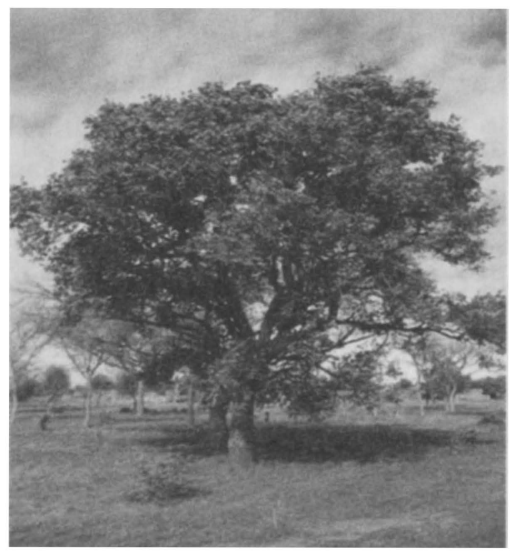

Figure 1: Detarium microcarpum dans un parc agroforestier au Mali.

Le présent article s'inscrit dans le cadre d'une valorisation plus raisonnée de $\mathrm{D}$. microcarpum qui est présentement très surexploitée. Il vise à analyser les pratiques paysannes sur le plan alimentaire et identifier les descripteurs biochimiques intéressants des fruits de $D$. microcarpum. Ce travail de recherche a été sous-tendu par les hypothèses suivantes: I) les populations rurales disposent de solides connaissances sur la collecte et les usages alimentaires des fruits de D. microcarpum; 2) la teneur en protéines, en sucre alimentaire et en vitamine $C$ des fruits de $D$. microcarpum peuvent orienter le choix des accessions pour les futurs programmes d'amélioration; et 3) un stockage de longue durée des fruits de D. microcarpum entraîne la baisse de leur teneur en vitamine $\mathrm{C}$.

\section{Méthodologie}

La méthodologie adoptée a consisté à faire une étude ethnobotanique, et une analyse physico-chimique et organoleptique des fruits au laboratoire.

\section{Accessions}

Les accessions maliennes de D. microcarpum concernées sont au nombre de six (tableau I).

\begin{tabular}{lll}
\hline Accession & Unité agro-climatique (I) & Sols (2) \\
\hline Lougourogombo & sahélien & $\begin{array}{l}\text { ferrugineux tropicaux lessivés à taches et } \\
\text { concrétions }\end{array}$ \\
\hline Soutè & sahélien & peu évolué sur cuirasse \\
\hline Néresso & nord soudanien & peu évolué sur grés \\
\hline
\end{tabular}




\begin{tabular}{lll}
\hline Accession & Unité agro-climatique (I) & Sols (2) \\
\hline Gouinso2 & sud soudanien & ferrugineux tropical lessivé induré sur cuirasse \\
\hline Bougoumbala & sud soudanien & ferrugineux tropical lessivé à taches et concrétions \\
\hline Oualia & sud soudanien & peu évolué sur cuirasse \\
\hline
\end{tabular}

(I) PIRT (1986); (2) Kéita (2002)

Tableau 1: Localisation agro-climatique des accessions maliennes de Detarium microcarpum.

\section{Enquête ethnobotanique}

L'enquête ethnobotanique a été réalisée au nord et au centre du sud du Mali pendant les mois d'août et septembre de l'an 20or. Cette zone couvre les cercles de Sikasso, Koutiala et Yorosso, où D. microcarpum est traditionnellement exploité par les communautés paysannes (carte). Ces villages ont été choisis en fonction de leur bonne connaissance de la gestion des ressources naturelles et de la présence d'une formation forestière naturelle à $\mathrm{D}$. microcarpum.

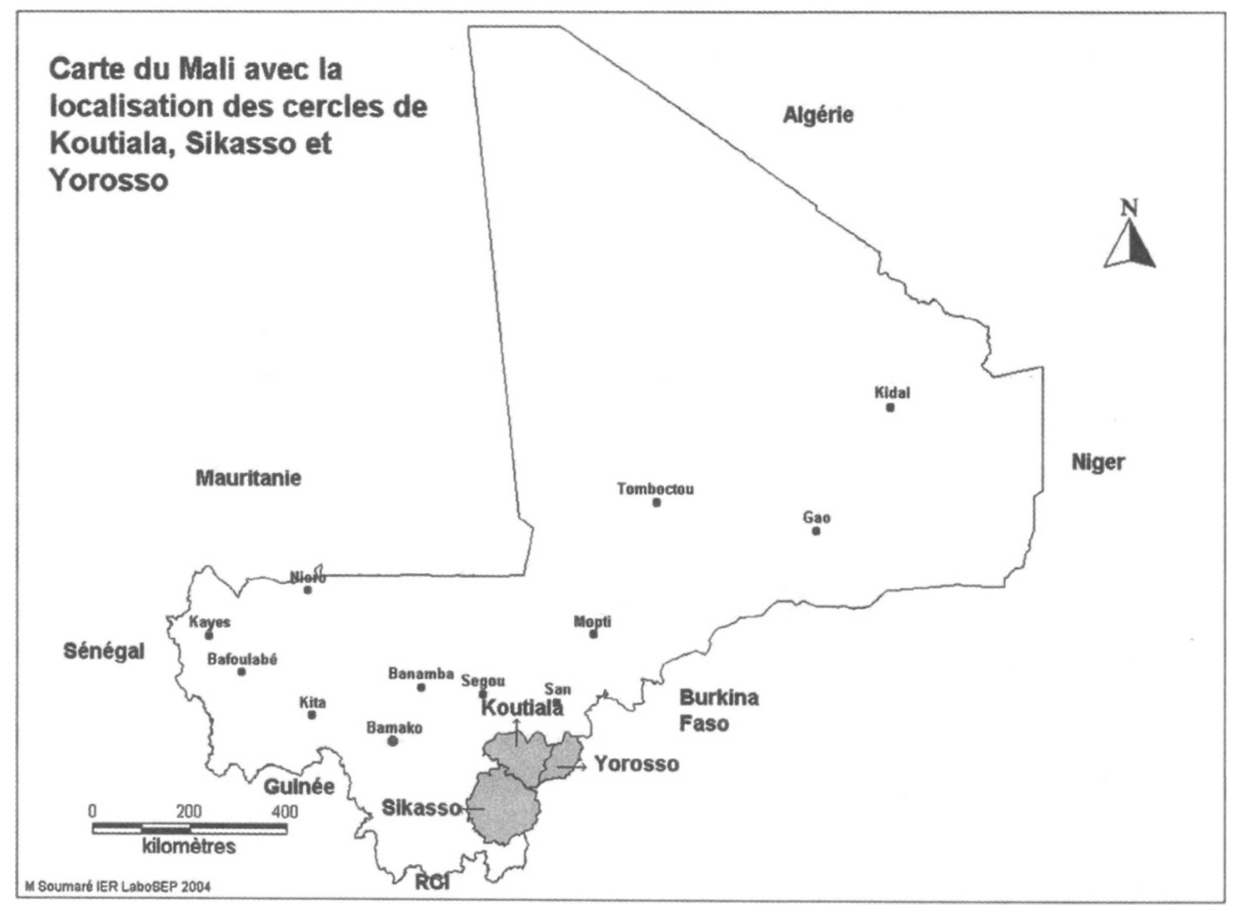

Carte 1: Localisation des sites.

L'enquête a été menée auprès de vingt villages dont trois villages sénoufo, quatorze villages minianka et trois villages bobofing. Elle a concerné un échantillon de 244 personnes dont ${ }_{4} 77$ hommes et 67 femmes. Les interviews semi-structurées par groupe ont été faites suivant la méthode décrite par Wentholt et al. (200I) qui consiste à interroger les femmes et les hommes séparément. Elles ont été faites en langue vernaculaire au 
moyen de questions ouvertes. Au niveau des trois ethnies, les questions ont porté sur les méthodes et techniques de cueillette, les personnes impliquées dans la cueillette, les organes utilisés dans l'alimentation humaine et le goût des fruits de D. microcarpum. Au niveau de chaque village, le choix des personnes à enquêter a été fait en fonction de leur disponibilité.

\section{Mesure des fruits}

La longueur $(\mathrm{mm})$ et la largeur $(\mathrm{mm})$ de chaque fruit ont été mesurées à l'aide d'un pied à coulisse avec une précision de $\pm 0,1 \mathrm{~mm}$. La longueur est prise du point d'attache du fruit à la branche jusqu'à l'extrémité du fruit. La largeur a été mesurée au niveau du plus grand diamètre du fruit (figure 2).

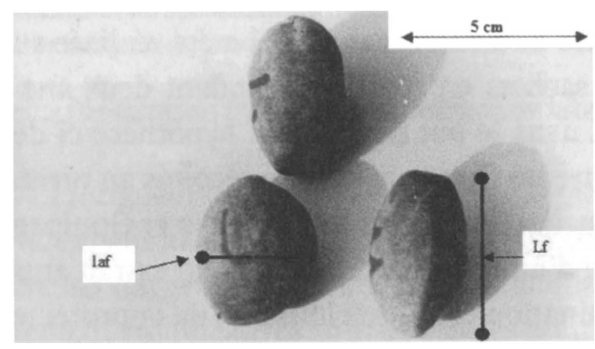

Figure 2: Mesure des dimensions des fruits de Detarium microcarpum récoltés au Mali. (Légende: Lf : longueur du fruit (mm) - laf: largeur du fruit (mm)).

\section{Test organoleptique}

Le test organoleptique a été initié pour formaliser les connaissances du groupe ethnique minianka au sud du Mali qui indique que des arbres de circonférence comprise entre $60 \mathrm{~cm}$ et $120 \mathrm{~cm}$ produisent des fruits sucrés (Kouyaté et al., 2002). Des fruits ont été récoltés à Néresso, Gouinso2 et Bougoumbala sur des arbres de petite taille $(20 \mathrm{~cm} \leq$ $\left.\mathrm{C}_{\mathrm{r}, 3 \mathrm{om}} \leq 60 \mathrm{~cm}\right)$ et de grande taille $\left(6 \mathrm{r} \mathrm{cm} \leq \mathrm{C}_{\mathrm{r}, 30 \mathrm{~m}} \leq \mathrm{I} 20 \mathrm{~cm}\right)$, à l'aide d'une perche munie d'un sécateur.

Le test organoleptique a été réalisé au laboratoire par dix personnes expérimentées et choisies au hasard. Il a porté sur le goût sucré comme facteur déterminant. Les notes ont été attribuées suivant une échelle hédonique utilisée par le Laboratoire de Technologie Alimentaire de l'Institut d'Economie Rurale au Mali: 7 points (très sucré), 6 points (sucré), 5 points (moyennement sucré), 4 points (légèrement sucré), 3 points (acide) et 2 points (amer).

\section{Evaluation biochimique}

L'évaluation biochimique a concerné un échantillon de trente fruits qui ont été cueillis sur quinze arbres de D. microcarpum par site, à raison de deux fruits et $20 \mathrm{~g}$ de pulpe par arbre. En 2003, les fruits ont été cueillis sur des arbres qui n'avaient pas été 
exploités, sains, en cours de production de fruits, et qui n'avaient pas été plantés par l'homme.

La pulpe a été extraite par simple frottage des fruits qui avaient été trempés dans l'eau distillée pendant 15 heures. Leur séchage à l'air ambiant a été effectué pendant quatre heures dans une chambre à $20^{\circ} \mathrm{C}$ de température. Le produit séché a été utilisé pour la détermination de la teneur en matière sèche et en protéines.

L'azote a été déterminé par la méthode de Kjeldhal. Cette méthode repose sur la combustion des échantillons frais avec l'acide sulfurique concentré pour permettre la réduction de l'azote organique à de l'azote minéral, en présence de catalyseur (Egan et al., I98I). Selon la même source, la protéine est déterminée en multipliant le poids total d'azote par le coefficient de transformation 6,25 qui est recommandé par la FAO pour tout genre d'aliments. La pulpe séchée dans une étuve à $105^{\circ} \mathrm{C}$ pendant 4 heures pour obtenir la matière sèche (Egan et al., 198I) a été pesée sur une balance Mettler (type $\mathrm{PE}$ $\mathrm{r} 60, \max =\mathrm{r} 60 \mathrm{~g}, \min =0,5 \mathrm{~g} \pm 0, \mathrm{I} \mathrm{g}$ ).

L'évaluation de la vitamine $\mathrm{C}$, du sucre alimentaire et du $\mathrm{pH}$ a été réalisée sur des pulpes qui ont été conservées dans des sachets en plastique pendant deux ans dans une chambre à une température de $20^{\circ} \mathrm{C}$ dans le but de vérifier l'hypothèse ci-dessus. Elle a été effectuée sur un échantillon de trente fruits qui ont été récoltés au niveau des accessions de Lougourogombo, Soutè, Oualia, Bougoumbala, Néresso et Gouinso2. Le sucre alimentaire a été déterminé, à l'aide d'un réfractomètre, dans un extrait aqueux à partir de la pulpe qui est purifiée par élimination et co-précipitation de la protéine avec du cyanure de zinc et du ferrocyanure de potassium en utilisant la méthode proposée par Southgate (I99I). Le pH de la pulpe a été déterminé à l'aide d'une électrode mise dans une solution aqueuse de $10 \%$ sans gaz carbonique $\left(\mathrm{CO}_{2}\right)$, en utilisant la méthode proposée par Egan et al. (I98I). La vitamine $\mathrm{C}$ a été déterminée par extraction de $\mathrm{H}_{3} \mathrm{PO}_{3}$ et par titration à l'indophénol en utilisant la méthode proposée par Egan et al. (I98I).

\section{Traitement et analyse des données}

Les données des enquêtes ethnobotaniques ont été traitées suivant la statistique descriptive en utilisant le taux de réponses. Ce choix est justifié par notre option d'enquêter par groupe. Le taux de réponses est le rapport entre le nombre de personnes qui ont répondu à une question et le nombre total de personnes présentes à l'assemblée (Van den Eyden et al., 1994; Cotton, 1996).

L'analyse de variance a été appliquée aux variables longueur, largeur et goût des fruits en utilisant le logiciel SPSS. Le test de Newman-Keuls a été appliqué pour faire la comparaison multiple des moyennes au seuil de $5 \%$.

Des corrélations entre les paramètres physico-chimiques et les dimensions des fruits ont été établies, à l'aide du logiciel SPSS. 


\section{Résultats}

Dimensions des fruits

\begin{tabular}{|c|c|c|c|c|}
\hline \multirow[t]{2}{*}{ Site } & \multicolumn{2}{|c|}{ Longueur des fruits (mm) } & \multicolumn{2}{|c|}{ Largeur des fruits (mm) } \\
\hline & Moyenne & CV (\%) & Moyenne & CV (\%) \\
\hline Lougourogombo & $39,0 \pm 3$, I a & 7,9 & $2 \mathrm{I}, 4 \pm 2, \mathrm{I}$ bc & 9,8 \\
\hline Soutè & $35,3 \pm 3,4 \mathrm{bc}$ & 9,6 & $22, I \pm I, 8 b$ & $8, \mathrm{I}$ \\
\hline Néresso & $32,0 \pm 3,3 d$ & 10,3 & $20,7 \pm I, 4 c$ & 6,8 \\
\hline Gouinso2 & $3^{6, I} \pm 7,5 \mathrm{~b}$ & 20,8 & $2 \mathrm{I}, 8 \pm 4,5 \mathrm{~b}$ & 20,6 \\
\hline Bougoumbala & $3^{6,9} \pm 2,7 b$ & 7,3 & $23,8 \pm 1,5$ a & 6,3 \\
\hline Oualia & $33,7 \pm 3,5 \mathrm{~cd}$ & IO, 4 & $22,0 \pm 1,7 b$ & 7,7 \\
\hline \multirow[t]{2}{*}{ Moyenne } & $35,45 \pm 4,00$ & II, 28 & $21,98 \pm 1,98$ & 9,00 \\
\hline & Probabilité & & Probabilité & \\
\hline Site & 0,000 & & 0,000 & \\
\hline
\end{tabular}

Les lettres indiquent des groupes de moyennes homogènes

Tableau 2: Dimensions des fruits de Detarium microcarpum suivant les accessions.

La longueur des fruits a varié de 32,0 $\mathrm{mm}$ (Néresso) à 39,0 mm (Lougourogombo). Les valeurs minimale et maximale de la largeur des fruits sont observées respectivement à Néresso (largeur $=20,7 \mathrm{~mm}$ ) et Bougoumbala (largeur $=23,8 \mathrm{~mm}$ ). Des différences hautement significatives sont observées pour les accessions de $\mathrm{D}$. microcarpum au seuil de $5 \%$. Les fruits de Lougourogombo sont plus longs que ceux des autres accessions, alors que de larges fruits sont rencontrés à Bougoumbala.

Le coefficient de variation de la longueur des fruits varie de $7,3 \%$ à $20,8 \%$, alors que celui de la largeur des fruits oscille ente $6,3 \%$ à $20,6 \%$. Ceci dénote que les accessions sont hétérogènes.

\section{Enquêtes ethnobotaniques}

Les résultats de l'enquête auprès des ethnies bobofing, minianka et sénoufo ont montré que la cueillette et le ramassage des fruits de D. microcarpum sont libres, c'est-à-dire qu'ils ne sont soumis à aucune autorisation du chef des terres. La cueillette est faite par les femmes et les enfants, soit en secouant les branches de l'arbre, soit en utilisant une gaule (figure 3). En ce qui concerne les parties de D. microcarpum utilisées dans l'alimentation humaine, les trois ethnies ignorent la consommation des feuilles, des racines, de l'écorce et des fleurs. Elles consomment exclusivement les fruits, soit à l'état crû, soit préparé. Les minianka utilisent les fruits dans la préparation des gâteaux et pour soigner la méningite. En milieu sénoufo ( $5 \%$ de réponses), les fruits sont utilisés dans la fabrication d'une boisson alcoolique et de couscous. Les fruits sont surtout consommés par les bobofing (33\% de réponses) pour traiter le paludisme et la diarrhée. Pour toutes ethnies confondues, les personnes interrogées ont noté que le goût des fruits est sucré. On peut dire que ces fruits, soumis à la cueillette, jouent à la fois un rôle alimentaire et médicinal. 


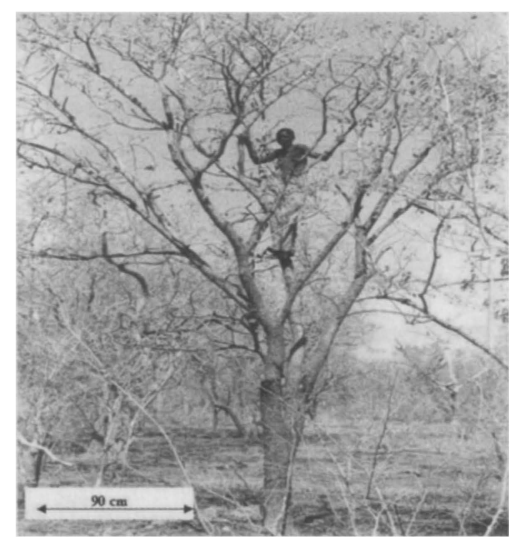

Figure 3: Récolte des fruits de Detarium microcarpum par un garçon au Mali. (Légende: Le garçon secoue les branches pour récupérer les fruits au niveau du sol).

\section{Test organoleptique}

\begin{tabular}{lllllll}
\hline Accession & Gouinso2 & \multicolumn{2}{l}{ Bougoumbala } & \multicolumn{2}{l}{ Néresso } \\
\hline Classe de circonférence à I,30 m (m) & $20-60$ & $6 \mathrm{I}-\mathrm{I} 20$ & $20-60$ & $6 \mathrm{I}-\mathrm{I} 20$ & $20-60$ & $6 \mathrm{I}-\mathrm{I} 20$ \\
\hline Dégustation & $5,2 \mathrm{ab}$ & $5,5 \mathrm{ab}$ & $5,6 \mathrm{ab}$ & $6,3 \mathrm{a}$ & $3,9 \mathrm{c}$ & $4,5 \mathrm{bc}$ \\
\hline Moyenne générale & & & $5, \mathrm{I} 67$ & & & \\
\hline
\end{tabular}

Les lettres indiquent des groupes de moyennes homogènes.

Tableau 3: Caractéristiques organoleptiques des fruits de Detarium microcarpum suivant les accessions.

Le goût des fruits de D. microcarpum cueillis au Mali a varié de 3,9 (légèrement sucré) à 6,3 (sucré). Les fruits des individus du sud soudanien (Bougoumbala et Gouinso2) sont significativement plus sucrés que ceux du nord soudanien (Néresso). En revanche, au niveau d'une accession donnée, le goût est significativement équivalent pour les fruits qui ont été cueillis sur des arbres de différentes tailles. Au regard de ces résultats, $\mathrm{D}$. microcarpum peut être classé parmi les espèces à fruit sucré.

\section{Caractéristiques physico-chimiques des fruits (tableaux 4 et 5)}

\begin{tabular}{lcc}
\hline Accession & Protéines $(\%)$ & Matière sèche $(\%)$ \\
\hline Néresso & 5,88 & 89,62 \\
\hline Gouinso2 & 4,70 & 89,6 I \\
\hline Bougoumbala & 4,29 & 88,14 \\
\hline Moyenne & 4,96 & $89, \mathbf{1 2}$ \\
\hline Ecart-type & 0,82 & 0,85 \\
\hline Coefficient de variation $(\%)$ & 16,53 & 0,95 \\
\hline
\end{tabular}

Tableau 4: Taux de protéines et de matière sèche des fruits de Detarium microcarpum . 
Le taux de matière sèche a atteint $89,12 \pm 0,85 \%$. Il a varié de $88,14 \%$ (Bougoumbala) à $89,62 \%$ (Néresso). Les protéines ont atteint $4,96 \pm 0,82 \%$. Les valeurs minimale et maximale enregistrées sont respectivement 4,29\% (Bougoumbala) et 5,88\% (Néresso).

\begin{tabular}{lccc}
\hline Accession & Sucre alimentaire (\%) & $\begin{array}{c}\text { Vitamine C } \\
\text { (mg/roo g de matière sèche) }\end{array}$ & pH \\
\hline Lougourogombo & 61,8 & 2,6 & 5,55 \\
\hline Soutè & 66,4 & 2,4 & 5,7 \\
\hline Néresso & 66,7 & 2,9 & 5,17 \\
\hline Gouinso2 & 63,2 & $4, \mathrm{I}$ & 4,87 \\
\hline Bougoumbala & 65,5 & 4,6 & 5,24 \\
\hline Oualia & 63,2 & 2,8 & 5,7 \\
\hline Moyenne & 64,5 & $\mathbf{3 , 2}$ & 5,4 \\
\hline Ecart - type & $\mathbf{2 , 0 0}$ & $\mathbf{0 , 9 0}$ & $\mathbf{0 , 3 3}$ \\
\hline Coefficient de variation (\%) & $\mathbf{3 , 1 0}$ & $\mathbf{2 8 , 1 2}$ & $\mathbf{6 , 1 1}$ \\
\hline
\end{tabular}

Tableau 5: Taux de sucre alimentaire, teneur en vitamine $\mathrm{Cet}$ pH des fruits de Detarium microcarpum suivant les accessions.

Les résultats des analyses au laboratoire ont montré que les fruits de D. microcarpum stockés pendant deux ans possèdent $64,5 \pm 2,00 \%$ de sucre alimentaire. Les valeurs minimale et maximale du sucre alimentaire ont atteint respectivement $6 \mathrm{r}, 8 \%$ à Lougourogombo et $66,7 \%$ à Néresso. La teneur en vitamine $\mathrm{C}$ a atteint en moyenne 3,2 \pm $0,9 \mathrm{mg} / \mathrm{Io0} \mathrm{g}$ de matière sèche. Elle a varié de $2,4 \mathrm{mg} / \mathrm{IO0} \mathrm{g}$ de matière sèche à Soutè à 4,6 $\mathrm{mg} / \mathrm{Ioo} \mathrm{g}$ de matière sèche à Bougoumbala, ce qui dénote une grande variabilité entre les accessions. Le $\mathrm{pH}$ a atteint 5,4 $\pm 0,33$. Les valeurs minimale et maximale sont observées respectivement à Gouinso2 $(\mathrm{pH}=4,87)$, et Oualia et Soutè $(\mathrm{pH}=5,7)$.

A l'exception de la vitamine $\mathrm{C}$, les coefficients de variation inter-accessions sont faibles pour le sucre alimentaire et le $\mathrm{pH}$.

Relation entre les caractéristiques physico-chimiques et les dimensions des fruits de Detarium microcarpum (tableau 6).

\begin{tabular}{lcccccc}
\cline { 2 - 7 } & Accession & Protéines & $\begin{array}{c}\text { Matière } \\
\text { sèche }\end{array}$ & $\begin{array}{c}\text { Sucre } \\
\text { alimentaire }\end{array}$ & Vitamine C & pH \\
\hline Accession & $\mathrm{I}$ & 0,96 & 0,98 & 0,34 & $-0,97$ & $-0, \mathrm{I} 8$ \\
\hline Protéines & 0,96 & $\mathrm{I}$ & 0,88 & 0,59 & $-0,99$ & 0,09 \\
\hline Matière sèche & 0,98 & 0,88 & $\mathrm{I}$ & $0, \mathrm{I} 3$ & $-0,90$ & $-0,38$ \\
\hline Sucre alimentaire & 0,34 & 0,59 & 0,13 & $\mathrm{I}$ & $-0,54$ & 0,87 \\
\hline Vitamine C & $-0,97$ & $-0,99$ & $-0,90$ & $-0,55$ & $\mathrm{I}$ & $-0,05$ \\
\hline p H & $-0,18$ & 0,09 & $-0,38$ & 0,87 & $-0,05$ & $\mathrm{I}$ \\
\hline Longueur fruit & $-0,93$ & $-0,99$ & $-0,83$ & $-0,65$ & 0,99 & $-0,19$ \\
\hline Epaisseur fruit & $-0,93$ & $-0,90$ & $-0,99$ & $-0,18$ & 0,92 & 0,34 \\
\hline
\end{tabular}

Les chiffres en gras indiquent les valeurs supérieures à $50 \%$.

Tableau 6: Corrélations entre les caractéristiques physico-chimiques et les dimensions des fruits de Detarium microcarpum. 
La matrice des corrélations entre les différentes variables montre que les accessions sont positivement et significativement corrélées avec le taux de matière sèche $(r=0,98)$ et de protéines $(r=0,96)$. Des corrélations significatives et négatives sont observées entre les accessions et les dimensions des fruits $(r=-0,93)$. A l'exception de la vitamine $\mathrm{C}(\mathrm{r}=0,92$ pour la largeur du fruit; $\mathrm{r}=0,99$ pour la longueur $\mathrm{du}$ fruit), les autres caractéristiques physico-chimiques sont négativement et significativement corrélées avec les dimensions des fruits.

\section{Discussion}

Les résultats de l'enquête ethnobotanique menée auprès des ethnies bobofing, minianka et sénoufo ont montré que, parmi tous les organes de D. microcarpum, seuls les fruits sont consommés au sud du Mali. L'utilisation des fruits dans l'alimentation humaine est rapportée par plusieurs auteurs (Arbonnier, 2000; Dakouo, 2002; Garine, 2002). La préparation de la bière locale à partir des fruits de D. microcarpum est pratiquée également par les populations des provinces de Sanmatenga et Zoundweogo au Burkina Faso (Wiersum et Slingerland, 1996).

En ce qui concerne le goût sucré des fruits, les résultats au laboratoire ont révélé que le goût n'est pas lié à la taille de l'arbre. Ceci ne corrobore pas la perception paysanne au sud du Mali qui stipule que les gros arbres donnent des fruits sucrés. Par ailleurs, ce goût sucré des fruits de D. microcarpum est signalé par plusieurs auteurs (Arbonnier, 2000; Boffa, 2000).

La teneur en vitamine $\mathrm{C}$ de 3,2 $\pm 0,9 \mathrm{mg} / \mathrm{Ioo} \mathrm{g}$ de matière sèche de nos échantillons de fruits est relativement bonne, même après deux ans de stockage. La grande variation de la teneur en vitamine $\mathrm{C}$ entre les accessions $(\mathrm{CV}=28, \mathrm{I} 2 \%)$ peut être attribuée surtout à la période de cueillette. La teneur mesurée au Sénégal est comprise entre o, o et 4,0 mg/100 g (Cavin, 2007), ce qui semble confirmer nos résultats. D'autres auteurs ont rapporté que les fruits frais ont une teneur en vitamine $C$ estimée à $32 \mathrm{mg} / \mathrm{Io0} \mathrm{g}$ de matière sèche (Lamien et al., 2008). Ceci indique que le stockage des fruits de D. microcarpum pendant deux ans a négativement affecté leur teneur en vitamine $C$. Les fruits de $D$. senegalense qui a été longtemps confondu à D. microcarpum, en contiennent 0,5 à I\% (Cavin, 2007). Cette confusion pourrait expliquer la remarquable teneur en vitamine $C$ attribuée aux fruits de $D$. microcarpum par plusieurs auteurs qui ne donnent par contre de valeur chiffrée (Alexandre, 2002; Vautier et al., 2007; Lamien-Meda et al., 2008).

On note une variation élevée entre les accessions pour les protéines, alors qu'elle est insignifiante pour la matière sèche. En ce qui concerne les protéines, les fruits de $D$. microcarpum récoltés au Mali ont un taux de 4,96 $\pm 0,8 \%$, ce qui confirme les résultats fournis par Boffa (2000).

Nos résultats ont montré que le taux de sucre alimentaire des fruits secs de $D$. microcarpum atteint $64,47 \%$. La comparaison entre $D$. microcarpum et d'autres espèces soudano-sahéliennes montre que les fruits de $\mathrm{D}$. microcarpum sont riches en sucre alimentaire. A titre d'exemples, les fruits secs de Boscia senegalensis, de Balanites aegyptiaca et de Ziziphus mauritiana ont un taux respectif de 6,6\%, 20,4 \% et 37,6\% en zone sahélienne 
nord du Sénégal (Nordeide, 1996; FAO, 2002; Danthu et al., 2002).

En ce qui concerne les corrélations, les résultats ont montré que les fruits de grande taille sont aussi les moins riches en protéines. Ils ont indiqué que le taux de sucre alimentaire varie suivant la longueur des fruits: plus le fruit est de petite taille, plus le taux de sucre alimentaire est élevé.

\section{Conclusion}

Les enquêtes ethnobotaniques ont mis en évidence l'utilisation des fruits de D. microcarpum dans l'alimentation humaine au niveau des populations rurales du sud malien. Ces fruits sont consommés à l'état crû ou préparé (gâteaux, couscous, bière locale), et ont un goût sucré.

L'évaluation des caractéristiques physico-chimiques des fruits de D. microcarpum au Mali a permis d'identifier des accessions dont les fruits sont riches en protéines et en sucre alimentaire (Nérésso), et en vitamine C (Bougoumbala et Gouinso2). Une variabilité au niveau de la taille des fruits a été observée entre les accessions.

La récolte des fruits de $\mathrm{D}$. microcarpum pour les futurs programmes d'amélioration doit être envisagée à Néresso, à Bougoumbala et à Gouinso2. A Néresso, elle concernera des petits fruits moins épais qui sont riches en protéines et en sucre alimentaire. A Bougoumbala et Gouinso2, l'accent sera mis sur la teneur en vitamine $C$ pour toute taille des fruits confondue. Il reste néanmoins à confirmer la raison génétique qui expliquerait ces différences.

\section{Références}

Alexandre, D. Y. (2002). Initiation à l'agroforesterie en zone sahelienne. Les arbres des champs du Plateau central au Burkina Faso. Khartala Editions, $220 \mathrm{p}$.

Arbonnier, M. (2000). Arbres, arbustes et lianes des zones sèches d'Afrique de l'Ouest. Cirad, MNHN, UICN, Montpellier, France. 54I p.

Boffa, J. M. (2000). Les parcs agroforestiers en Afrique subsaharienne. Cahier FAO, Conservation 34: 42-190.

Cavin, A. L. (2007). Contribution à la connaissance taxonomique et chimique de fruits africains du genre Detarium (Fabaceae - Caesalpinioideae): D. microcarpum Guill. et Perr. et des formes comestibles et toxiques de D. senegalense, J.F. Gmel. Thèse de Doctorat, Univ. Génève, Suisse. 277 p.

Cotton, C. M. (1996). Ethnobotany. Principles and Applications. John Wiley \&Sons, 424 p.

Dakouo, J. M. (2002). Impact de l'aménagement participatif sur la végétation des forêts de la zone soudanienne nord du Mali. Cas de la forêt classée de Dioforongo (Ségou). Thèse de doctorat, Université de droit, d'économie et des sciences d'Aix-Marseille, Aix-Marseille III, 175 p.

Danthu, P., Soloviev, P., Totté, A., Tine, E., Ayessou, N., Gaye, A. \& Niang, T. D. (2002). Caractères physico-chimiques et organoleptiques comparés de jujubes sauvages et des fruits de la variété Gola introduite au Sénégal. Fruits, 173-182.

Egan, H., Kirk, R. S. \& Sawyer, R. (1981). Pearson's chemical analysis of foods. Eighth edition, Churchill Livingstone, 2-21.

Freiberger, C. E., VanderJagt, D. J., Pastuszyn, A., Glew, R. S., Mounkaila, G., Millson, M. \& Glew, R. H. (1998). Nutrient content of the edible leaves of seven wild plants from Niger. Plant Food Hum. Nutr. 53: pp. 57-69.

Garine, I. de. (2002). Nourriture de brousse chez les Muzey et les Masa du Nord-Cameroun. Méga, Tchad. 13 p. 
Glew, R. S., Dorothy, J., Chuang, L.-T., Huang, Y.-S., Millson, M. \& Glew, R. H. (2005). Nutrient content of four edible wild plants from West Africa. Plant Foods Hum. Nutr., 60: 187-193.

Kéita, B. (2002). Les sols dominants du Mali. In: FAO. (eds.) Quatorzième réunion du sous-comité ouest et centre africain de corrélation des sols pour la mise en valeur des terres. Abomey-Calavi, Bénin, 9-13 oct. 12 p.

Kouyaté, A. M. (2005). Aspects ethnobotaniques et étude de la variabilité morphologique, biochimique et phénologique de Detarium microcarpum Guill. \& Perr. au Mali. Thèse de Doctorat. Faculté des Sciences Biologiques Appliquées. Université de Gand. Belgique. 207 p.

Lamien-Meda, A., Lamien, C. E., Compaoré, M. M. Y., Meda, R. N. T., Kiendrebeogo, M., Zeba, B., Millogo, J. F. \& Nacoulma, O. G. (2008). Polyphenol content and antioxydant acitivty of fourteen wild edible fruits from Burkina Faso. Molecules, 13: 58I-594.

Lamien, N., Lingani-Coulibaly, P. \& Traoré-Gue, J. (2008). Importance of local fruits consumption in diets balance in Burkina Faso, West Africa. 7 p. (www.icuc-iwmi.org/symposium2008/2-3-Lamien. pdf)

Nordeide, M. B., Hatloy, A., Folling, M., Lied, E. \& Oshaug, A. (1996). Nutrient composition and nutritional importance of green leaves and wild food resources in an agricultural district Koutiala in southern Mali. Int. J. Food Sc. and Nut., 47: 455-468.

PIRT (1986). Zonage agro-écologique du Mali. Commission consultative nationale d'évaluation et de suivi de la mise en œuvre de la stratégie alimentaire. Groupe zonage écologique et adéquation des projets. CCE/USAID. I5I $\mathrm{p}$.

Sène, E. H. (2000). Forêts et sécurité alimentaire en Afrique. La place de la foresterie dans le Programme spécial pour la sécurité alimentaire de la FAO. In: FAO (eds.). Forêts, sécurité alimentaire et moyens de subsistance durables.

Southgate, D. A. T. (1991). Determination of Food Carbohydrates. 2ed. Elsevier applied science. IX. $232 \mathrm{p}$.

Van den Eynden, V., Van Damme, P. \& De Wolf, J. (1994). Inventaire et modelage de la gestion du couvert végétal pérenne dans une zone forestière du sud du Sénégal. Rapport final. Partie $\mathrm{C}$ Etude ethnobotanique. Université de Gand, Belgique, 33-99.

Vautier, H., Sanon, M. $\beta$ Sacande, M. (2007). Detarium microcarpum Guill. \& Perr. Seed leaflet, $n^{\circ} 122$.

Wentholt, W., Dembélé, A. R. K. \& Diallo, M. (200I). Genre et recherche agricole au Mali. IER. KIT Publishers. The Netherlands. I4I p.

Wiersum, K. F. \& Slingerland, M. (1996). Use and management of two multipurpose tree species (Parkia biglobosa and Detarium microcarpum) in agrosylvopastoral land-use systems in Burkina Faso. Wageningen, Pays-Bas, $29 \mathrm{p}$. 